American J. of Engineering and Applied Sciences 3 (1): 214-221, 2010

ISSN 1941-7020

(C) 2010 Science Publications

\title{
Fiber Optic Sensors for Transportation Infrastructural Health Monitoring
}

\author{
Chin Chang and Rahul Mehta \\ Department of Electrical Engineering, California State University, Long Beach, CA 90840, USA
}

\begin{abstract}
Problem statement: Monitoring the health of transportation infrastructures is currently reliant on transportation maintenance teams. Scheduled and periodic inspections on most of the infrastructures are performed by manual and visual operations, which are generally time consuming and costly procedures. The use of the fiber optic sensor technology makes it possible to realize continuous, real time and automatic health monitoring for the transportation infrastructure. Approach: This article provided a tutorial introduction to transportation infrastructural health monitoring using fiber optic sensors. Since the topic spans many disciplines, our goal was limited to providing a basic conceptual framework. We began by reviewing the prerequisite topics from structural health monitoring and fiber optic sensors, including a brief review of point and distributed fiber optic sensor technologies. We then provided a comprehensive review of key fiber optic sensors which may be used in the transportation infrastructure monitoring. Results: Point fiber optic sensors were first discussed and then distributed fiber optic sensors. Performances of each sensor are discussed based on the transportation infrastructure monitoring application. Conclusion: We concluded the tutorial with a number of observations on the current directions of the research field of the fiber optic sensor for the transportation infrastructure health monitoring.
\end{abstract}

Key words: Fiber optic sensor, transportation infrastructure, health monitoring

\section{INTRODUCTION}

The collapse of the Minneapolis Bridge has raised a public safety issue on the nation's 73,784 bridges rated "structurally deficient" by the Department of Transportation (Arnoldy, 2007). The improvement of the bridge and overpass safety has become a strong motivating factor, in particular, after the accidental collapse of the Minneapolis Bridge. Knowing the integrity of in-service transportation infrastructures on a continuous, real-time basis is a very important objective for transportation maintenance teams. The economic motivation is another essential factor to consider for adapting viable technology to achieve ultimate public safety need. A cost-effective and innovative technology is imperative for effectively monitoring and managing health of bridges and overpasses without increasing a substantial tax burden to all tax payers.

Monitoring the health of transportation infrastructures is currently reliant on transportation maintenance teams. Scheduled and periodic inspections on most of the infrastructures are performed by manual and visual operations, which are generally time consuming and costly procedures. Recently, Johnson (2003) of the University of
Southern California proposed an approach for bridge structural health monitoring based on variable stiffness and damping devices. This approach offers the potential to provide more accurate parametric changes required for bridge health monitoring.

The use of the fiber optic sensor technology makes it possible to realize continuous, real-time and automatic health monitoring for the transportation infrastructure. The technology offers several advantages such as: Minimized downtime, avoidance of catastrophic failure and reduction in maintenance labor. In addition, any new design of the infrastructure may encompass fiber optic sensors to make smart structures. With well-developed fiber optic networking technology, a remote laser source may be used as a signal source to accurately detect parametric changes at different locations of the structure. The detected data may be recorded and analyzed in order to obtain precise structural information on the infrastructure. In summary, the realization of fiber optic sensor monitoring system for the transportation infrastructure may greatly improve public safety with low operation costs.

Given the current interest in health monitoring and management for the transportation infrastructure

Corresponding Author: Chin Chang, Department of Electrical Engineering, California State University, Long Beach, CA 90840, USA Tel: 562-985-5150 
systems utilizing fiber optic sensor technology, it seems both appropriate and timely to provide a tutorial introduction to this topic. Our objective is to assist others in transportation infrastructural health monitoring and management systems based on fiber optic sensors by providing a consistent terminology and nomenclature and an appreciation of possible applications.

To assist newcomers to the field we will structure the paper as follows. We first review the relevant fundamentals of health monitoring and management of transportation infrastructures. A comprehensive review of key fiber optic sensor technology will be then given with emphasis of infrastructural parameter monitoring. Finally, we present a number of deployments of fiber sensing systems for transportation infrastructures.

Background: Structural Health Monitoring (SHM) is a process to measure key physical and chemical parameters relevant to the structure condition. Engineers use the measured data to detect damage and determine the location, type and extent of the damage. The damage detection enables a maintenance team to implement preventive plan to correct the structural deficiency and thus enhance public safety and avoid catastrophic disaster. They may also estimate the lifetime span of the structure based on the measured data from the SHM system. On the other hand, as a new infrastructure is under the construction, engineers may employ the SHM system to gather structural data for design verification, quality control and infrastructure rating and risk assessment.

A typical SHM system, as illustrated in Fig. 1, is composed of a network of sensors that measure the parameters related to the state of the structure and its environment. For transportation infrastructures such as bridges, overpasses and tunnels, the most important parameters are: Positions, deformations, strains, pressures, accelerations and vibrations. In addition, chemical parameters such as humidity, $\mathrm{pH}$ value and chlorine concentration are almost as important as those physical parameters. Environmental factors often have substantial impact on the condition and operation of the transportation infrastructure. Those environmental parameters of interest are temperature, wind speed and direction, solar irradiation, precipitation, snow accumulation, water level and flow. The SHM system might be additionally equipped with a wireless network for remote monitoring, control and alarm for effective system operations.

Conventional sensors based on electro-mechanical transducers are currently used for measuring most of the physical and environmental parameters mentioned previously. Recently, fiber optic sensors have begun to enter into the SHM system. Fiber optic sensor technology offers enormous advantages and capabilities compared to its counterparts in the SHM application. Not mention its economic impact, its ability to provide real-time, continuous structural parameter measurement is already beyond that of the conventional sensor. The fiber optic sensors are electrical passive and can be easily networked together to form a passive sensor network system for SHM applications. Taking the advantage of the widely deployed fiber optic communication networks, remote structural mentoring and management systems may be readily implemented for almost all transportation infrastructures in the nation. Therefore, the public safety will be ensured and the maintenance cost for the transportation infrastructure will be significantly reduced.

\section{MATERIALS AND METHODS}

Fiber optic sensors: The research and development of the fiber optic technology has inspired revolutionary changes to our lives today. The technological advances in fiber optics have paved the way for the widely deployed fiber optic communication networks, well known as the information super highway. Today, we heavily rely on fiber optic networks to transmit and receive data, voice and video signals via the internet (Brackett, 1989; Barry, 1989).

The fiber optic sensor technology may be considered as an extension of the fiber optic communication technology. It has been commonly used in various applications including industrial automation, health care, aerospace and aviations. Compared to the conventional sensor technology, the fiber optic sensor offers numerous advantages such as high sensitivity, all solid state, no moving parts and a long lifetime.

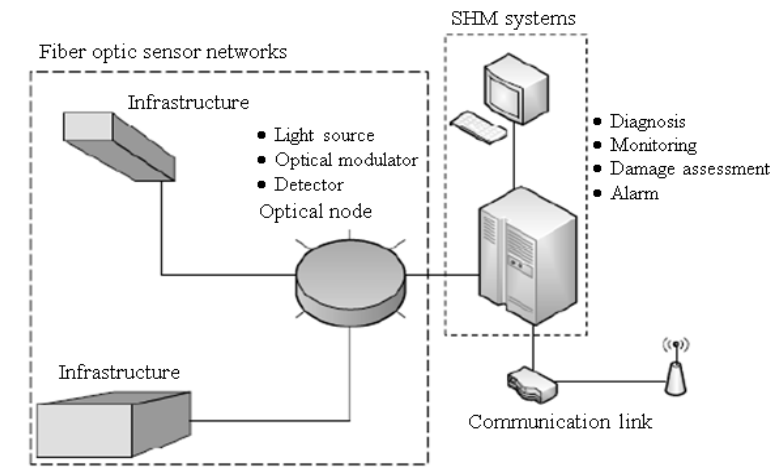

Fig. 1: Schematic of structural health monitoring and management system 
Here, we will explore several key fiber optic sensors which are essential to the SHM applications. In addition to the advantages mentioned previously, fiber optic sensors are capable of performing continuous, real-time structural parameter measurement which is impractical if we adapt the conventional sensors (Frangopol et al., 2008). All fiber optic sensors considered for the transportation infrastructural health monitoring are passive devices; thus no power system is required at the location of the structure. In a SHM system, optical fiber may also be used as transmission medium to transport measured signals form optical fiber sensor networks which are deployed in the infrastructure; hence remote monitoring is feasible.

In larger perspective, applications of fiber optic sensors in the infrastructure health monitoring and management may be classified into two categories: (i) structural performance monitoring and (ii) surveillance applications. Sensitivity requirement for performance monitoring sensors are typically medium to low for parameter measurement, while surveillance sensors require ultra high sensitivity to the measurement parameter. Performance monitoring sensors are typically used for: (i) monitoring the strain profile of large structures (e.g., bridge or a ship hull), (ii) monitoring and tracking crucial parameters (e.g., temperature, pressure, or acceleration) at crucial locations of a given system and (iii) vibration monitoring for system identification and damage location. Since a majority of fiber sensors for performance monitoring relies on measuring the strain in the fiber, either directly in the case of strain sensing or indirectly in the case of fiber optic transducers, we will address sensor requirements in terms of strain resolution. Resolution requirements for performance strain monitoring range from a few ne to tens of $m \varepsilon$, depending upon the details of the application. Bandwidth requirement can vary from static (DC) to tens of $\mathrm{kHz}$.

Surveillance sensors, on the other hand, are exploited for measuring very faint signals and require sensors with strain resolution in the order $10^{-13}-10^{-14}$ and a frequency response from tens of $\mathrm{Hz}$ to tens of kHz. A good example of a high performance surveillance sensor is the fiber optic hydrophone, used for underwater surveillance (Davis et al., 1997). Strain resolution of the order of $10^{-14}$ is difficult to achieve with fiber Bragg grating and until recently surveillance sensors have been the exclusive realm of fiber interferometric sensors. However, fiber Bragg Grating Laser Sensors (FBGLs) have recently demonstrated strain resolution of $10^{-14}$ (Koo and Kersey, 1995), thus opening up the possibility of marking novel surveillance sensors.

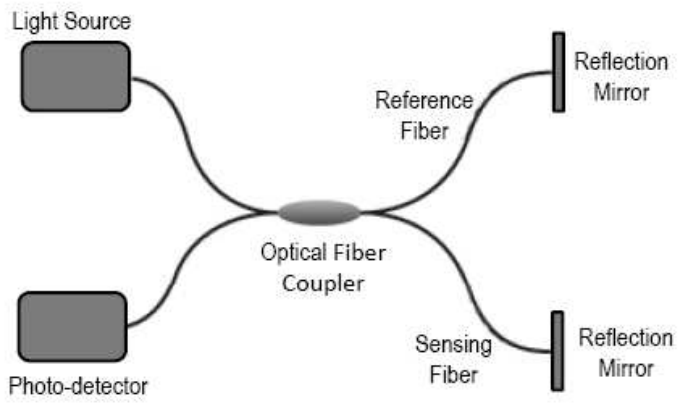

Fig. 2: Fiber optic Michelson interferometer

Most common structural parameters that fiber optic sensors measure are temperature, strain, pressure, deformation, vibration and acceleration. The SHM system based on the fiber optic sensor network may obtain structural parameter measurement of high sensitivity and accuracy for those parameters. Here, we will provide a review for several major fiber optic sensors to be used in the SHM applications.

Michelson interferometer sensors: The fiber optic Michelson interferometer consists of a single-mode fiber directional coupler with reflection mirrors formed on the cleaved ends of both fibers on the same side of the coupler (Kashyap and Nayar, 1983) as depicted in Fig. 2. As a sensor, one of the fibers is used as a reference fiber and the other is used to sense structural parameter. Any deformation in a transportation infrastructure will result in a change in the length difference of the two fibers. The interference between the two reflected light beams produces interference fringes. The shift of the fringe is an indirect measurement of the deformation strain.

To obtain accurate and reliable measurement of the deformation strain, a second mechanically scanning Michelson interferometer will be applied at the input/output port of the first fiber-optic interferometer to directly measure the length difference instead of the shift of the fringe pattern. The scanning Michelson interferometer is to create an optical path length difference such that it is equal to the length difference of the two fibers. Furthermore, a broadband light source or white light source having a low coherent length has to be used for the robust measurement. The scanning Michelson interferometer can accurately measure the optical path length difference by balancing the change of the length difference of the two fibers and hence yields the direct measurement deformation strain.

The fiber optic sensor based upon Michelson interferometer is a long-gauge sensor for deformation 
strain measurement with a resolution in the range of micrometers. It has excellent long term stability and is insensitive to the temperature. The schematic of the sensor for measuring structural deformation strain is illustrated in Fig. 3.

Fiber Bragg grating sensors: Since the discovery of photosensitivity in optical fibers in 1978 by Hill et al. (1978) and the subsequent demonstration of holographically-written gratings in fibers by Meltz et al. (1989), significant progress has been made towards the realization of various types of gratings in optical fibers. Advances in grating fabrication methods and fiber photosensitivity enhancement techniques have made it possible to fabricate a variety of index-modulated structures within the core of an optical fiber including Bragg grating (López-Higuera, 2002), long period gratings (Vengsarkar et al., 1996), $\pi$-phase shifted grating (Canning and Seats, 1994), blazed or tilted gratings (Erdogan and Sipe, 1995) and various types of chirped gratings (Byron et al., 1993).

Fiber grating devices have seen a wide variety of applications in both telecommunication and sensor fields. Fiber Bragg Gratings (FBGs) have been routinely used with semiconductor lasers for producing stable single frequency light signal with which extreme broadband communication link may be realized. They also play an important role in reconfigurable optical networks in the future optical network systems, while it is clear that grating-based devices are seeing great commercial success in the SHM applications. Transducers using FBGs such as pressure and acceleration sensors also have various applications and now become commercially available. Requirements and designs of several reported grating-based transducers used in SHM applications will be discussed.

A fiber grating is made by periodically changing the refractive index in the glass core of the fiber. The refractive index changes are achieved by exposing the fiber to the UV-light with a fixed pattern. The schematic of a fiber optic grating is illustrated in Fig. 4.

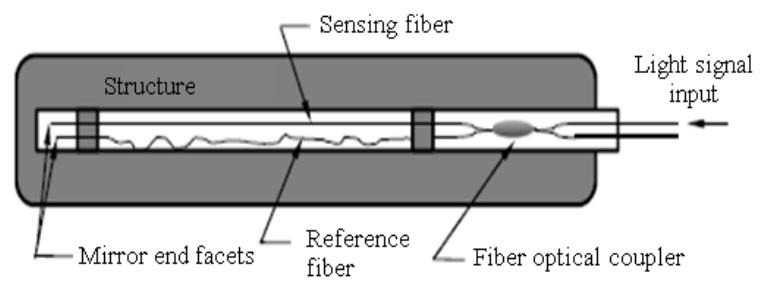

Fig. 3: A long-gauge deformation sensor using fiber optic Michelson interferometer

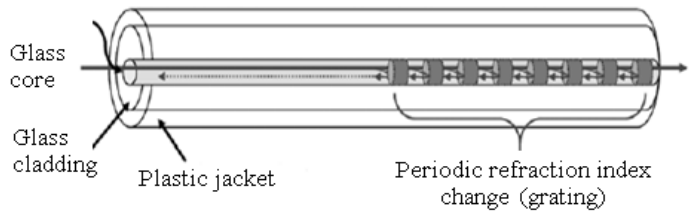

Fig. 4: Schematic of a fiber Bragg grating

As the input light propagates through the grating having a period of $\Lambda$ and the optical wavelength $\lambda$ satisfies the Bragg condition; the maximum reflection occurs. The Bragg condition may be expressed as:

$\lambda=2 \mathrm{n}_{\mathrm{eff}} \Lambda$

where, $\mathrm{n}_{\text {eff }}$ is the effective index of refraction of the optical fiber. The reflection and transmission spectra are shown in Fig. 5. Since $\mathrm{n}_{\text {eff }}$ is determined by the refractive index profit of the fiber; thus, it changes as the variations of ambient temperature and external mechanical stress. As the result, the center frequencies of the reflected and transmitted spectra will shift accordingly. Therefore, the measured frequency shift is an indication of the temperature and strain changes. FBGs can accurately measure local temperature and strain in a small region of the infrastructure with very high sensitivity.

A summary of potential sensing applications of various types of fiber gratings is provided in Table 1. The chart is not necessarily meant to be comprehensive and to cover all conceivable application areas in fiber grating sensors. Of course, there is no one 'magic' fiber optic sensor which covers the full range of sensing requirements for all applications. In this paper we only briefly discuss FBG applications and examples from various field tests involving such sensors.

Table 1: Summary of potential sensing applications of various types of fiber gratings

\begin{tabular}{ll}
\hline Grating type & Applications \\
\hline Fiber Bragg Grating & Strain and temperature sensors \\
(FBGs) & Pressure sensors \\
& Acceleration sensors \\
& Ultrasound sensors \\
& Mechanical load sensors \\
& Gas detection sensors \\
& Extensometer \\
& Electromagnetic field sensors \\
& Reflection elements in interferometric \\
& sensor arrays \\
Fiber Bragg Grating Laser & Novel, compact hydrophones \\
sensors (FBGLs) & Acoustic emission sensor for NDE \\
Long Period Gratings & Bend sensors \\
(LPGs) & Chemical sensors \\
& Broadband source filters \\
Pi Phase shifted gratings & Transverse load sensing \\
Chirp gratings & Strain sensing \\
& FBG demodulation \\
\hline
\end{tabular}


Am. J. Engg. \& Applied Sci., 3 (1): 214-221, 2010

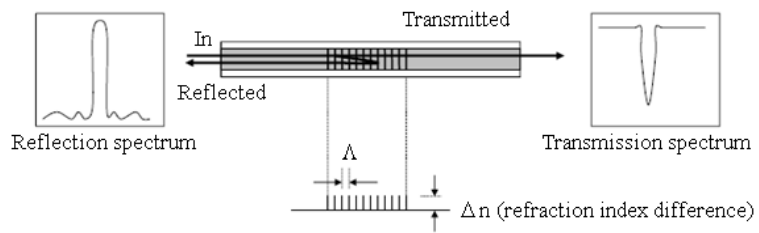

Fig. 5: Reflection and transmission spectra of a FBG

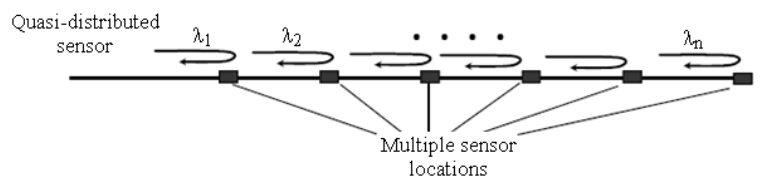

Fig. 6: Quasi-distributed sensor using an array of FBG sensors

Particular emphasis will be given to multiplexed networks based on FBGs for quasi-distributed measurements of parameters such as load, strain, temperature, and vibration. Observation has indicated that in certain cases the technology is fairly well developed and ready for widespread commercialization.

An array of FBGs has great potential for providing high performance structural sensing systems. Such measurements provide useful information regarding verification of novel construction approaches, infrastructure load rating systems and history of large loading events. Figure 6 depicts the schematic of a distributed sensor array used for SHM systems. In fact, the distributed array of FBG sensors is a quasidistributed sensor array, since the FBG sensor is a point sensor. This quasi-distributed senor array may be deployed over the entire transportation infrastructure to monitor strains and temperature at various locations. As previously discussed, this type of senor has very high resolution and accuracy of strain measurement; may be in the order of ne.

Although the quasi-distributed FBG sensor has very good performance in strain measurement, it cannot distinguish strain and temperature as the effective index $\mathrm{n}_{\mathrm{eff}}$ is dependent of both strain and temperature. Hence, a separated distributed temperature sensor is needed in order to extract the strain information for the system.

Distributed fiber sensors: Distributed fiber optic sensors are important sensor technologies which may be used in the infrastructure health monitoring for large and long structures such as bridges, beams and clamping ropes, from hundreds of meters to more than ten kilometers of length. Conventional sensor technology is often not feasible for the real-time and continuous structural parameters monitoring in these infrastructures (Frangopol et al., 2008). Based on the light scattering in the optical fiber, the distributed fiberoptic sensor is capable of continuously monitoring major structural parameters and has high spatial resolution, in the order of a few centimeters; hence, it allows structure engineers to measure structural parameters in an entire infrastructure system for design verification and damage and reliability assessments.

\section{RESULTS AND DISCUSSION}

There are several methods available for extracting distributed strain and temperature information from optical fiber. These include techniques based on Rayleigh, Raman and Brillouin scattering. Rayleigh scattering is the elastic scattering of light by particles much smaller than the wavelength of the light. It may occur when light wave travels in optical fiber therein the refractive index along the fiber fluctuates. Rayleigh light is scattered in all directions from the spatial variation of refractive index along the optical fiber. The intensity of the scattered light at each location along the fiber is sensitive to both local strain and temperature. By measuring the back scattered light with coherent signal detection method, one can readily extract the average strain and temperature information within a small segment of the fiber. Fiber optic strain sensor having a gauge length of less than $0.5 \mathrm{~m}$ and a strain sensitivity of less than $\mathrm{n} \varepsilon / \sqrt{\mathrm{Hz}}$ at $2 \mathrm{kHz}$ based on Rayleigh backscatter using a Time Division Multiplexing (TDM) scheme has been demonstrated (Posey et al., 2000). Sang et al. (2008) present a technique based on measuring the spectral shift of the intrinsic Rayleigh backscatter signal along the optical fiber and converting the spectral shift to temperature. Using Optical Frequency Domain Reflectometry (OFDR) to record the coherent Rayleigh scatter pattern results in spatial resolution of around $1 \mathrm{~cm}$ and provides temperature measurement with an accuracy of $0.6 \%$ of full scale temperature up to $850^{\circ} \mathrm{C}$. Although a distributed stain and/or temperature sensor with small gauge length and high measurement accuracy may be obtained based on Rayleigh scattering, it requires a separate sensor to either calibrate temperature for measuring strain at a particular location or vice versa.

Raman- and Brillouin-scattering phenomena have been used for distributed sensing applications over the past few years. Raman scattering was first proposed for sensing applications in the 1980s, whereas Brillouinscattering was introduced for strain and/or temperature monitoring applications very recently. Both Ramanand Brillouin-scattering effects are associated with 
different dynamic inhomogeneities in the silica and, therefore, have completely different spectral characteristics.

Raman scattered light is caused by thermally activated molecular vibrations. Because the scattered light is generated by thermal agitation, one may expects a frequency shift from the incident light wave. As the incident light slightly losses its energy to the molecular, this process, referred to Stokes scattering, leads to the scattered light with lower optical frequency. On contrary, the light wave gaining energy from the molecular produces the scattered light with a slightly higher frequency is referred to as anti-Stokes scattering. It is found that the amplitude of the anti-Stokes component is strongly temperature dependent, whereas, that of the Stokes component is not. Hence, optical signal processing technique utilizing Stokes and antiStokes components is needed to realize distributed temperature sensors. The magnitude of the anti-Stokes Raman scattering light is about one thousand times smaller $(-30 \mathrm{~dB})$ than that of the Rayleigh scattered light; multimode optical fiber is commonly exploited in the sensing system for improved scattered light capture. However, the attenuation coefficient of the multimode fiber is high; therefore, this technique limits the maximum measurement range up to $8 \mathrm{~km}$ only.

Brillouin scattering results from the scattering of light by sound waves. Thermally excited acoustic waves (acoustic phonons) produce a periodic modulation of the refractive index. Brillouin scattering occurs when light is diffracted backward on this moving grating, giving rise to frequency shifted Stokes and anti-Stokes components. For intense beams (e.g., laser light) travelling in an optical fiber, the variations in the electric field of the beam itself may produce acoustic vibrations in the medium via electrostriction. The beam may undergo Brillouin scattering from these vibrations, usually in opposite direction to the incoming beam, a phenomenon known as Stimulated Brillouin Scattering (SBS). The working principle of SBS may be schematically illustrated in Fig. 7. The Brillouin frequency shift attributed to the strain and temperature may be express as:

$v_{B}=v_{B o}+B_{T}\left(T-T_{o}\right)+B_{S}\left(\varepsilon-\varepsilon_{o}\right)$

where, $v_{\mathrm{Bo}}$ and $v_{\mathrm{B}}$ is the peak frequency of the SBS for unstrained and strained fibers, respectively. In Eq. 2, $\mathrm{B}_{\mathrm{T}}$ and $B_{S}$ are Brillouin thermal and strain co efficiencies.

The distributed sensor based on SBS is most suited for SHM applications. The distributed optical fiber Brillouin sensor based on the SBS has been investigated for pipeline buckling and concrete/FRP column monitoring (Bao et al., 2006). Using coherent detection scheme, the SBS-based sensor offers several advantages of long measured length, high resolution and accuracy and stability. The most important feature of the SBS-based Brillouin distributed sensor is its ability to measure strain and temperature separately or simultaneously with high spatial resolution and accuracy.

Figure 8 (Nikle's et al., 1997) shows the experiment results of the temperature characteristics of the SBS distributed sensor. The Brillouin frequency shift in an order of tens of $\mathrm{GHz}$ exhibits a fairly linear relation with the measure temperature as in Fig. 8a. On contrary, as can be shown in Fig. 8b, the Brillouin linewidth decreases when the measured temperature increases. The experimental results in Fig. 9a show that the Brillouin frequency shift also linearly depends on the fiber elongation which is the indication of the local strain; however, the linewidth is independent of the elongation, as shown in Fig. 9b. These measurements conclude that the temperature and strain may be measured separately base on Brillouin frequency shift and linewidth variation.

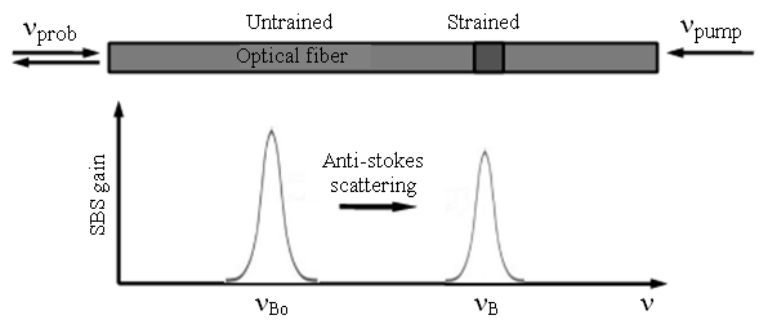

Fig. 7: Stimulated Brillouin Scattering in single mode optical fiber
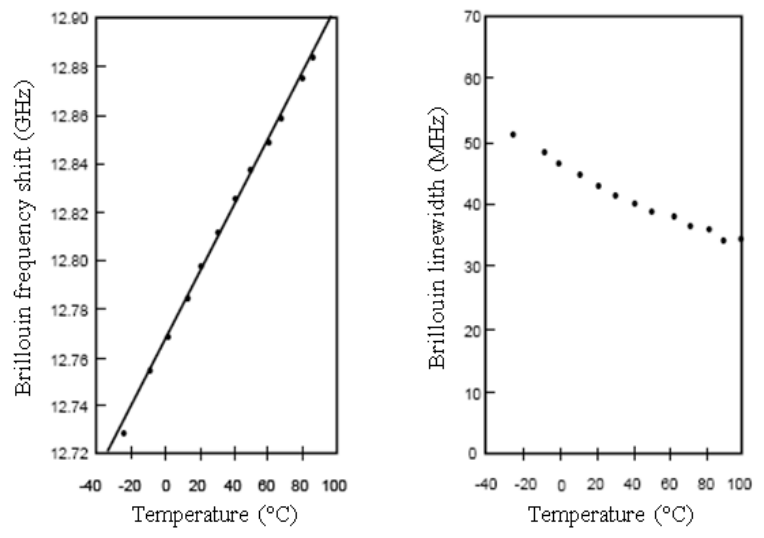

Fig. 8: Temperature characteristic of SBS distributed sensor 

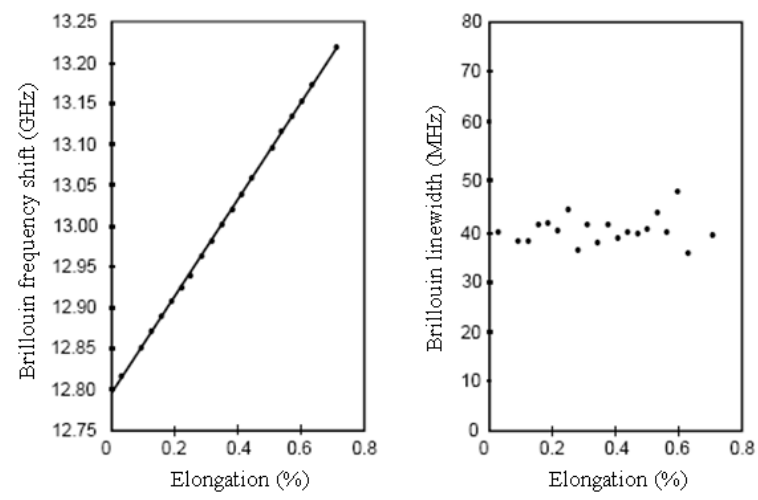

Fig. 9: Spectrum characteristic of SBS distributed sensor

The Brillouin distributed sensor technology allows fast measurement of strain and temperature to be achieved within a few seconds owing to the use of straight forward optical signal processing technique. Furthermore, the monitoring system may incorporate optical amplifier to booth up the optical power to extend the measurement range; this makes the Brillouin distributed sensor a practical choice for the transportation infrastructure monitoring system.

\section{CONCLUSION}

Majority of structural monitoring sensors used in long span bridge health monitoring systems are still those based on conventional transducer technology. Akashi Kaikyo Bridge in Japan, world's longest suspension bridge, uses seismometer, anemometer, accelerometer, velocity gauge, Global Positioning System (GPS), girder edge displacement gauge, Tuned Mass Damper (TMD) displacement gauge and thermometer for dynamic monitoring (Sumitro et al., 2001). Significant progress in the development of fiber optic sensors and wireless sensors has been made in the past decade and some of them are now commercially available. Fiber optic sensors have successfully been applied for the long term SHM of large scale bridges such as Confederation Bridge (Cheung et al., 1997) and Tsing Ma Bridge (Lau et al., 1999), whereas wireless sensors for the bridge SHM application is still in the technology demonstration stage.

Some conventional sensors currently used in long span bridge health monitoring are deficient in providing adequate accuracy and long term stability. For example, a GPS used in Akashi Kaikyo Bridge for absolute displacement or deflection monitoring has three limitations: (i) the measurement accuracy of a GPS is not good enough to completely meet bridge health monitoring requirement; (ii) a GPS does not work well for monitoring the displacement of piers beneath the bridge deck (caused by ships colliding, settlement) and (iii) a GPS is not capable to accurately measure the deflection in the foggy environment. A long gauge deformation sensor using the fiber optic Michelson interferometer discussed previously becomes an ideal choice for the application because of its long term stability and capability of measuring the absolute displacement.

Fiber optic sensors such as FBG and SBS sensors are only applied to the measurement of strain and temperature with high sensitivity and accuracy; one may used to implement a quasi-distributed sensor network and the other is a distributed sensor. Fiber optic sensors have additional advantages of low bias drift and high frequency response in addition to those mentioned early. Electrical gauges for strain and vibration monitoring normally encounter technical problems in either bias stability and/or dynamic response where fiber optic sensors may be the solutions.

Fiber optic sensor technology for the SHM application is still not widely accepted for the transportation infrastructure monitoring. The research and development in the fiber optic sensor seems to focus on measurements in strain, deformation and temperature; other key structural parameter monitoring is still lack of research and development effort. In order to take full advantage of the technology, research and development effort has to be directed into other parameter monitoring applications.

\section{ACKNOWLEDGEMENT}

The researchers and specially Chin Chang are very grateful to the support by the METRANS Transportation Center. METRANS is dedicated to solving metropolitan transportation problems through research, education and outreach.

\section{REFERENCES}

Arnoldy, B., 2007. How to pay for US road and bridge repair? The Christian Science Monitor. http://www.csmonitor.com/2007/0810/p03s01usec.html?page $=1$

Barry, R.A., 1989. IEEE Network: Special Issue on Optical Networks. 10.

Bao, X., F. Ravet and L. Zou, 2006. Distributed brillouin sensor based on brillouin scattering for structural health monitoring. Proceeding of the Optical Fiber Communication Conference, 2006 and the 2006 National Fiber Optic Engineers Conference, Mar. 5-10, IEEE Xplore Press, Anaheim, CA., pp: 3. DOI: 10.1109/OFC.2006.215419 
Brackett, C.A., 1989. Special issue on lightwave systems and components. IEEE communication Magazine: 27.

Byron, K., K. Sugden, T. Bircheno and I. Bennion, 1993. Fabrication of chirped Bragg gratings in photosensitive fibers. Elect. Lett., 1659-1660.

Canning, J. and M.G. Seats, 1994. $\pi$-phase shifted periodic distributed structures in optical UV post processing. Elect. Lett., 30: 1344-1345. http://ieeexplore.ieee.org/xpl/freeabs_all.jsp?tp=\&a rnumber $=311947 \&$ isnumber $=7568$

Cheung, M.S., G.S. Tadros, T. Brown, W.H. Dilger and A. Ghali et al., 1997. Field monitoring and research on performance of the confederation bridge. Can. J. Civil Eng., 24: 951-962. DOI: 10.1139/cjce-24-6-951

Davis, A.R., C.K. Kirkendall, A. Dandridge and A.D. Kersey, 1997. 64 channel all optical deployable acoustic arrays. Proceeding of the 12th Conference Optical Fiber Sensors, pp: 616. http://www.opticsinfobase.org/abstract.cfm?URI= OFS-1997-OFA6

Erdogan, T. and J.E. Sipe, 1995. Radiation mode coupling loss in tilted fiber phase grating. Opt. Lett., 20: $1838-1840$. http://www.opticsinfobase.org/abstract.cfm?id=346 13

Frangopol, D.A., A. Strauss and S. Kim, 2008. Bridge reliability assessment based on monitoring. ASCE J. Bridge Eng., 13: 258-270. http://cedb.asce.org/cgi/WWWdisplay.cgi?163798

Hill, K.O., Y. Fujii, D.C. Johnson and B.S. Kawasaki, 1978. Photosensitivity in optical fiber waveguides: Application to reflection filter fabrication. Appl. Phys. Lett., 32: 647-649. DOI: 10.1063/1.89881

Johnson, E.A., 2003. Innovative bridge structural health monitoring using variable stiffness and damping devices. METRANS Research Project, Project Number 03-17.

http://www.metrans.org/research/2003/03-17.htm

Kashyap, R. and B. Nayar, 1983. An all single-mode fiber Michelson interferometer sensor. J. Lightwave Technol., 1: 619-624. http://ieeexplore.ieee.org/xpl/freeabs_all.jsp?arnum ber $=1072153$

Koo, K.P. and A.D. Kersey, 1995. Bragg grating based laser sensor systems with interferometric interrogation and wavelength division multiplexing. J. Lightwave Technol., 13: 1243-1249. DOI: $10.1109 / 50.400692$
Lau, C.K., W.P.N. Mak, K.Y. Wong, W.Y.K. Chan and K.L.D. Man, 1999. Structural Health Monitoring of Three Cable-Supported Bridges in Hong Kong. Structural Health Monitoring, Chang, F.K. (Ed.). Lancaster (PA), Technomic, pp: 450-460.

López-Higuera, J.M., 2002. Handbook of Optical Fiber Sensing Technology. Wiley, ISBN: 10: 0471820539, pp: 828.

Meltz, G., W.W. Morey and W.H. Glenn, 1989. Formation of Bragg gratings in optical fibers by a transverse holographic method. Opt. Lett., 14: 823-825. DOI: 10.1364/OL.14.000823

Nikle`s, M., L. Th'evenaz and P.A. Robert, 1997. Brillouin gain spectrum characterization in singlemode optical fibers. J. Lightwave Technol., 15: 1842-1851. DOI: 10.1109/50.633570

Posey, R. Jr., G.A. Johnson and S.T. Vohra, 2000. Strain sensing based on coherent Rayleigh scattering in an optical fiber. Elect. Lett., 36: 1688-1689. DOI: 10.1049/el:20001200

Sang, A.K., M.E. Froggatt, D.K. Gifford, S.T. Kreger and B.D. Dickerson, 2008. One centimeter spatial resolution temperature measurements in a nuclear reactor using Rayleigh scatter in optical fiber. IEEE Sensors J., 8: 1375-1380. DOI: 10.1109/JSEN.2008.927247

Sumitro, S., Y. Matsui, M. Kono, T. Okamoto and K. Fujii, 2001. Long span bridge health monitoring system in Japan. Proc. SPIE., 4337: 517-524. http://adsabs.harvard.edu/abs/2001SPIE.4337..517S

Vengsarkar, A.M., P.J. Lemaire, J.B. Judkins, V. Bhatia, T. Erdogan and J.E. Sipe, 1996. Long-period fiber gratings as band-rejection filters. J. Lightwave Technol., 14: 58-65. DOI: 10.1109/50.476137 